\title{
ENSEÑAR MATEMÁTICA CON UN ENFOQUE HISTÓRICO EN LA ESCUELA SECUNDARIA
}

\author{
Por María Celeste Vazquez: \\ Universidad del Noroeste de la Provincia de Buenos Aires, Argentina. \\ m-celevazquez@hotmail.com \\ Natalia Fátima Sgreccia ${ }^{* \star}$ \\ Universidad Nacional de Rosario, Argentina. \\ nataliasgreccia@hotmail.com
}

Recibido: 29/09/2016 Aceptado: 22/12/2016

\begin{abstract}
Resumen
Esta investigación surge de inquietudes sobre la enseñanza de los números irracionales en cuarto año de la educación secundaria. La insatisfacción que los alumnos manifiestan durante el aprendizaje de este campo numérico y el desconocimiento de estos números luego de haberse impartido su enseñanza incentivaron a diseñar una propuesta didáctica basada en un enfoque histórico. La intención fue caracterizar la manera en que los alumnos desde la acción y reflexión construyen sus propios conocimientos mediante este tipo de enfoque. También interesó reconocer aspectos favorables y desfavorables de la experiencia. La teoría de referencia se delimitó desde cuatro perspectivas: histórica, educativa, matemática y curricular, que a su vez sirvieron de sostén metodológico. Se analizó la información recabada de observaciones de clases, de producciones de los estudiantes y de registros del docente. Los hallazgos parecen indicar que aprender los números irracionales a través de un enfoque histórico contribuye a los aprendizajes.
\end{abstract}

Palabras clave: Historia de la Matemática - Números irracionales - Escuela secundaria.

\section{Abstract}

This research stems from concerns about the teaching of the irrational numbers in the fourth year of secondary school. The students expressed dissatisfaction during learning of this numeric field and the ignorance of these numbers after having given its teaching encouraged to designing a didactical proposal based on an historical approach. The intent was to characterize how students from the action and reflection build their own knowledge through this approach. Also we interested to recognize favorable and unfavorable aspects of the experience. The theory of reference was delimited from four perspectives: historical, educational, mathematical and curricular, which in turn served as methodological support. Information gathered from classroom observations, student productions and teacher records were analyzed. The findings suggest that learning the irrational numbers through an historical approach contributes to learning.

Keywords: History of Mathematics - Irrationalnumbers - Secondaryschool.

\section{Presentación}

A través del tiempo que llevamos en nuestra tarea docente hemos observado en el estudio de la Matemática la dificultad que los alumnos manifiestan al aprenderla, la incomprensión que creen tener al respecto y la poca aplicabilidad que ellos le encuentran a algunos contenidos específicos de esta área del conocimiento.

Actualmente se observa una gran insatisfacción en el quehacer matemático de los alumnos de la Escuela Secundaria Agraria de la ciudad de Pergamino (Argentina). Esto se refleja en el interrogatorio que los alumnos manifiestan en el aula con respecto a la funcionalidad de la Matemática en la vida cotidiana. Ellos han expresado en muchos de los diálogos compartidos que esta área del conocimiento fue creada por "locos que estaban aburridos y no tenían nada para hacer", experimentando así sentimientos negativos con respecto a su aprendizaje.

\footnotetext{
Profesora de Tercer Ciclo de la EGB y de la Educación Polimodal en Matemática y Licenciada en Enseñanza de la Matemática. Se desempeña como docente en la Universidad del Noroeste de la Provincia de Buenos Aires así como en varias escuelas secundarias de Pergamino.

$2^{2^{* *}}$ Profesora de Enseñanza Media y Superior en Matemática, Magíster en Didácticas Específicas mención Matemática y Doctora en Humanidades y Artes mención Ciencias de la Educación. Se desempeña como docente en el Profesorado en Matemática de la Facultad de Ciencias Exactas, Ingeniería y Agrimensura de la Universidad Nacional de Rosario.
} 
Este malestar que manifiestan los alumnos impacta en su desempeño escolar, en el que suelen estudiar cada unidad didáctica separada del resto; estudian para el momento en que se los evalúa y luego al necesitar de conocimientos anteriores parece que todo ha sido olvidado. Muchas veces esta situación viene de la mano de que cuando estudian no les encuentran sentido a algunos contenidos matemáticos. Un ejemplo claro se refleja cuando los alumnos deben aprender sobre el campo de los números irracionales, debido a que no le ven aplicabilidad como a los otros campos numéricos.

Estas tensiones manifiestas en los procesos de aprendizaje a su vez por lo general se relacionan con modos de configuración de los procesos de enseñanza de la Matemática en la Escuela Secundaria. A diferencia de otras materias escolares, la Matemática suele enseñarse sin una contextualización de sus contenidos. Por ejemplo, se estudian los números irracionales, pero no todo el proceso vivido para dar con este conocimiento o la respuesta que el mismo le dio a una necesidad de determinada época o circunstancia.

Consideramos que enseñar Matemática con su Historia puede enriquecer el desarrollo de nuevas habilidades en los alumnos, al descubrir que "esos locos que no tenían nada que hacer" dieron respuestas a muchas necesidades que han ido surgiendo en la Historia de la humanidad y a su vez fueron abriendo nuevas puertas hacia el conocimiento, contribuyendo así a la generación de más y más dilemas por resolver.

Atendiendo a estas inquietudes desarrollamos una propuesta didáctica para el aprendizaje de los números irracionales que incorpora aspectos históricos y la implementamos en cuarto año de la Escuela Agraria de la ciudad de Pergamino, con el fin de analizar su incidencia en el aprendizaje de este campo numérico. Básicamente indagamos:

¿Cómo construyen los alumnos el significado de número irracional cuando las clases se desarrollan siguiendo una propuesta didáctica con un enfoque histórico?

¿Cuáles son los aspectos favorables y desfavorables de la propuesta didáctica para que los alumnos construyan el significado de número irracional?

Partimos del supuesto que aprender los números irracionales a través de un enfoque histórico contribuye a desarrollar en los estudiantes un espíritu crítico, que les permita valorar la importancia de este campo numérico y la significatividad de los mismos.

\section{Fundamentación}

Mirar la Matemática desde otra perspectiva, para no presentarla como un "edificio imponente" que en muchas ocasiones lo que hace es agobiar a nuestros alumnos más que interesarlos, puede lograrse acompañándola con su Historia (Wheeler, 1980). De hecho, la Historia de la Matemática puede constituirse en fuente de inspiración, autoformación y orientación en la actividad docente (González, 2004).

La introducción del aspecto histórico-epistemológico de la construcción del conocimiento matemático en la enseñanza no ubica al alumno en una posición pasiva de receptor de verdades acabadas, ya que se presenta el contenido desde una posición más cercana a su construcción, desde sus limitaciones, marchas y contramarchas. Enseñar Matemática con su Historia puede mejorar la actitud de los alumnos hacia esta área del conocimiento, permitiendo interpretar los acontecimientos históricos, mostrando cómo se utilizaban ciertos conocimientos en ciertas circunstancias. De esta manera se alimentarían justificaciones de la faz utilitaria de la Matemática y se posibilitaría a que los alumnos vayan teniendo idea de las evoluciones de la cimentación del conocimiento científico, al saber que los resultados y procedimientos asociados no fueron siempre como los conocemos hoy (Lara y Sgreccia, 2010).

Un cierto conocimiento de la Historia de la Matemática debería formar parte del bagaje de conocimientos del matemático, en general, y del profesor de cualquier nivel. En el caso de este último, no solo con la intención de que lo pueda utilizar como instrumento en su propia enseñanza, sino porque la Historia le puede proporcionar una visión verdaderamente humana de la ciencia y de la Matemática (de Guzmán, 1992; Jorge, Paixão y Cabrita, 2010). De hecho, en la comunidad internacional de investigación en Didáctica de la Matemática, existe una línea denominada "Historia y Educación Matemática" (Gómez, 2011) requiriéndose mayor claridad y consenso sobre los aportes de la Historia a la Educación Matemática (Anacona, 2003) así como vinculaciones en la clase diaria (Sorando, 2005).

Por lo controversial de su Historia, resulta de especial interés el aprendizaje de los números irracionales. El conocimiento de las operaciones y sus propiedades se requiere para temas muy importantes (Teorema de Pitágoras, distancia entre dos puntos, ecuación ordinaria de la circunferencia, cálculo de áreas, la resolvente, sustituciones trigonométricas, entre tantos otros donde se involucren modelos matemáticos continuos). Sumando a esto, los números reales constituyen la base del análisis matemático y del desarrollo del pensamiento numérico-algebraico. Autores como Sánchez y Valdivé (2014) reconocen como problema didáctico al aprendizaje del concepto de número, en particular irracional.

El Diseño Curricular jurisdiccional de cuarto año del secundario (Dirección General de Cultura y Educación de la Provincia de Buenos Aires, 2009) propende a que el alumno interprete que $\sqrt{2}$ es un número y no una operación a resolver. También sugiere que el trabajo de los alumnos no sea mecánico. Desde la propuesta didáctica deseamos avanzar invitando a los estudiantes a construir su propio conocimiento matemático específico de manera significativa, involucrando la Historia y generando una participación activa en las clases.

A continuación se presentan las cuatro perspectivas teóricas del estudio: histórica, educativa, matemática y curricular. 


\section{Perspectiva histórica}

Los pitagóricos consideraban que el gran sistema del mundo, el ser, la forma y la acción de todas las cosas son una consecuencia natural de los números. Estos determinan el nexo de unión de todos los entes y la mecánica del universo entero, son la base del espíritu y el único medio por el cual se manifiesta la realidad. Al número 1 lo concebían como el principio y la causa de todo. Era la verdadera esencia de todas las cosas y el fundamento de cuanto existe. Para los pitagóricos todo procedía de la unidad y a ella se podía reconducir todo. El orden pitagórico se basaba en la finitud. Toda pluralidad como fruto de la unidad es finita, está formada de números enteros y también toda fragmentación o división de la unidad procede de ellos y por tanto de la unidad.

La grandeza sublime del Teorema de Pitágoras y la mágica belleza del Pentagrama místico pitagórico fueron dos de los tópicos más relevantes de la Escuela pitagórica, pero se convirtieron en dos caballos de Troya para la Geometría griega, porque llevaban en su interior el germen de la profunda crisis de la comunidad pitagórica donde aparecieron. Los Diálogos de Platón informan que la comunidad matemática griega se vio gravemente sofocada por un descubrimiento que prácticamente demolía la base de la fe pitagórica en los números enteros. Las consecuencias de su principal teorema atentaron contra los fundamentos de su doctrina, que les había llevado a establecer un paralelismo entre el concepto numérico y la representación geométrica. La diagonal del cuadrado no era conmensurable con el lado; igualmente sucedía en el pentágono regular tan emblemático para los pitagóricos. La creencia de que los números podían medirlo todo era una simple ilusión y así quedaba eliminada de la Geometría la posibilidad de medir siempre con exactitud.

Se había descubierto la magnitud inconmensurable, lo irracional, elalogon, que provocaría una crisis sin precedentes en la Historia de la Matemática (Boyer, 1986). Esta crisis involucró tres nociones matemáticas: el número y su concepción entera, finita y positiva; el principio de no contradicción que propició el rechazo de los irracionales al conllevar a la paridad de los números impares como consecuencia de suponer la diagonal conmensurable; el campo operativo donde se realizan las operaciones por estar inmerso el número. Se analizó el concepto de número y en el siglo XIX se desarrolló una teoría para los irracionales, cuando Dedekind (1831-1916) introdujo las "cortaduras" para realizar la construcción formal de los números reales.

El descubrimiento de la inconmensurabilidad marcó un hito en la Historia de la Geometría, porque no es algo empírico, sino puramente teórico. Su aparición señaló el momento más dramático no solo de la Geometría pitagórica sino de toda la Geometría griega y fue con seguridad lo que imprimió a la Matemática griega un cambio de rumbo que la convertiría en la obra de ingeniería geométrico-deductiva plasmada en Los Elementos de Euclides.

Como consecuencia de la aparición de las magnitudes inconmensurables, los griegos no podían reconocer la existencia de números irracionales, lo que les dificultaba el tratamiento numérico de longitudes, áreas, volúmenes y ángulos. El abismo que se había abierto entre número y magnitud continua impedía someter las magnitudes geométricas a manipulaciones algebraicas, lo que determinó la transformación del Álgebra oriental que los pitagóricos habían heredado de los babilonios en el Álgebra geométricadel Libro II de Los Elementos de Euclides, en la que los números son sustituidos por segmentos de recta y las operaciones entre ellos se llevan a cabo mediante construcciones geométricas. Con gran habilidad en la práctica geométrica, los griegos hicieron de su Álgebra geométricaun poderoso instrumento para la resolución de ecuaciones, mediante el método de la Aplicaciónde las áreas (Babini, 1966).

Cuando la Matemática griega comenzó a declinar, Diofanto (200-284) abandonó la representación geométrica de los números y empezó a desarrollar las reglas del Álgebra y la Aritmética, utilizando un literal para representar las incógnitas de una ecuación. En esta etapa, Europa se estancó científicamente y el desarrollo matemático se desplazó hacia la India, Asia Central y los países árabes, impulsándose sobre todo la Astronomía.

Fueron los indios, entre los siglos $\mathrm{V}-\mathrm{XV}$, los que inventaron el sistema de numeración actual, introdujeron los números negativos y comenzaron a operar con los números irracionales de forma semejante que con los racionales sin representarlos geométricamente. Es importante resaltar que el conocimiento de los números por parte de los griegos no fue superado hasta 24 siglos más tarde. Los matemáticos Cantor (1845-1918), Dedekind (1831-1916), Weierstrass (1815-1897) y Bolzano (1781-1848) fueron los que culminaron la obra, que duró medio siglo de investigaciones, sobre los números naturales, enteros, racionales e irracionales, que considerados juntos, constituyeron lo que se denominó el sistema de los números reales.

\section{Perspectiva educativa}

Esta investigación toma como sustento para su fundamentación a la Educación Matemática Crítica (EMC) (Skovsmose, 1999; Valero, Andrade-Molina y Montecino, 2015), debido a que la base de esta corriente se contrapone al enfoque mecanicista de la enseñanza de la Matemática al mismo tiempo que es propulsora de un cambio en la enseñanza tradicional de esta área. Los educadores críticos respaldan la didáctica crítica, en donde se asume una relación activa entre autorreflexión y acción, y se estudia el currículo desde un enfoque emancipador, que implica la adquisición de autonomía frente a estereotipos de pensamiento.

La década de 1980 marcó un tiempo importante en la preocupación de la comunidad de Educación Matemática 
internacional en cuanto a la incidencia de la Matemática en la sociedad y su desarrollo. El $5^{\circ}$ Congreso Internacional de Educación Matemática, que se realizó en Australia en 1984, privilegió el porqué y para qué enseñar Matemática, dando importancia a la relación Matemática-Educación-Sociedad y discutiendo sobre la necesidad de un nuevo rol social para la Educación Matemática en un mundo en el que la tecnología desempeña un papel importante.

En los inicios de esta década de cambios profundos en la concepción de la Educación Matemática, Higginson (1980) la visualizó en términos de interacciones de las caras de un tetraedro en donde la Matemática, la Psicología, la Sociología y la Filosofía constituyen disciplinas fundacionales de la Educación Matemática.

Una década después de presentado el modelo de Higginson surgió otro modelo dentro de la concepción, creado por Steiner (1990), y que incluyó ciencias referenciales planteadas en el modelo anterior (Matemática, Historia de la Matemática, Psicología, Sociología, Pedagogía) y abrió una nueva perspectiva, al incluir todo el sistema social relacionado con la comunicación de la Matemática, en el que identificó nuevas áreas de interés para la Educación Matemática, como la problemática del "nuevo aprendizaje de la sociedad", ligado al uso de las tecnologías de la información y comunicación (TIC).

En esta búsqueda de modelos que expliquen la complejidad de la Educación Matemática y su incidencia en el desarrollo social de los pueblos, se encuentra en los inicios del siglo XXI el modelo creado por el investigador venezolano Mora (2005). Coloca un bloque de disciplinas básicas referenciales, en donde muestra coincidencias con el modelo tetraédrico de Higginson y con el sistémico de Steiner, así como disciplinas objeto de la enseñanza y el aprendizaje, donde sitúa a la Matemática y a la Historia de la Matemática, entre otras. Lo innovador de esta propuesta es la visión integral de la Didáctica de la Matemática, como un encuentro entre el hombre y la Matemática. El autor reivindica el papel de los docentes de Matemática, en todos los niveles de enseñanza, y las construcciones que realizan junto con sus alumnos día a día, advirtiendo sobre el peligro de haber convertido al ser humano en una simple máquina manipuladora y consumidora de conocimientos, en nuestro caso matemáticos, sin reflexionar sobre sus consecuencias, importancia social y política.

La Teoría Crítica ha influido en el aprendizaje y la enseñanza de la Matemática, al constituirse la EMC. Esta asume algunos de sus constructos para ser teorizados y aplicados en la práctica pedagógica del profesor en Matemática o en otros contextos en los que se manejen conocimientos matemáticos. Se destacan entre ellos: la educación dialógica y problematizadora, la reflexión y acción, la emancipación, la competencia democrática, el conocimiento reflexivo matemático, la relación Cultura-Matemática, la Matemática como construcción humana y social, el docente-alumno como sujetos políticos y no solo cognitivos. El análisis de estos constructos debe ayudar a los docentes no solo a una reflexión práctica sobre el conocimiento didáctico del contenido a enseñar, sino a indagar críticamente sobre cómo sus acciones pedagógicas tienen repercusiones morales y éticas en los estudiantes.

Desde esta perspectiva de la EMC, es necesario que los implicados en el aprendizaje y la enseñanza de la Matemática asuman una actitud de autorreflexión, de crítica, y a la vez puedan descubrir y transformar las relaciones de poder subyacentes en las prácticas matemáticas y pedagógicas, tanto de alumnos como de docentes e instituciones educativas. El conocimiento matemático se presenta en varias ocasiones como un saber instrumental fuertemente conectado con un simbolismo bien estructurado, lo que ha hecho que los implicados en su aprendizaje no hayan captado ni participado en la reconstrucción y construcción de ese conocimiento, y quizás tampoco en la reflexión de cómo ese conocimiento puede ayudarlos a reinterpretar su mundo de vida.

Una influencia recibida en la EMC es la de Freire (2000), quien considera al diálogo como un elemento fundamental, pues a través del mismo tanto el educador como el educando se apropian del mundo, de la realidad en la cual viven para actuar sobre ella y transformarla y humanizarla, es decir, desarrolla en ambos una conciencia crítica. Esta relación dialógica genera en ambos, mediante la reflexión crítica del contenido y el currículo, una conciencia de su propia realidad y su transformación. Además, el aprendizaje y la enseñanza de la Matemática no son considerados "neutros", pues sus contenidos responden a intereses ideológicos, políticos, económicos y culturales, los cuales deben ser explicitados y problematizados por los involucrados a través del diálogo, la reflexión y la crítica.

La perspectiva histórica señala no solo los saberes matemáticos acumulados por el ser humano, y fruto de los cuales la ciencia moderna se ha originado, sino una revisión sobre cómo se sitúa la Matemática en la experiencia de cada uno y del colectivo.

\section{Perspectiva matemática}

Un número real puede ser un racional o irracional. Los números racionales son aquellos que pueden expresarse como el cociente de dos números enteros, mientras que los irracionales son los que no pueden expresarse de ese modo. Los números racionales también pueden describirse como aquellos cuya representación decimal es eventualmente periódica, mientras que los irracionales tienen una expansión decimal aperiódica. Hay valores que no se pueden expresar como números racionales y estos son los irracionales, tal es el caso de $\sqrt{2}$. Este tipo de números introdujo en el mundo pitagórico la emergencia del inconmensurable como ser que se escapa al dominio de la unidad, ya que ninguna operación entera con él es capaz de retornarlo al origen de todo que es la unidad o mónada pitagórica. Al inconmensurable se lo llama irracional porque no se puede expresar como razón de dos enteros, pero sobre todo por ser 
algo ininteligible, que está fuera del logos (alogon), es decir, representa la sinrazón (Bell, 1985).

\section{Perspectiva curricular}

Según el Diseño Curricular de tercer año del nivel secundario, los alumnos deben acceder a la noción de número irracional en segundo año con miras al reconocimiento de su existencia y a su diferenciación con los números racionales. Además propone que en tercer año se integre este contenido con los del eje Geometría y Magnitudes, ya que los números irracionales aparecieron en la Historia de la Matemática vinculados a la geometría y las mediciones. Se sugiere que el docente elabore una secuencia para el trabajo de sus alumnos en la que tengan la oportunidad de calcular las medidas de las diagonales de diferentes cuadrados y rectángulos utilizando el teorema de Pitágoras, explorando la representación gráfica de los mismos para abordar las nociones de segmentos conmensurables e inconmensurables, de completitud de la recta cuando en ella se incorporan los números irracionales y nombrar al conjunto numéricocomo el de los números reales. También se encomienda que estas nociones se continúen profundizando en los últimos años de la escolaridad secundaria.

En cuanto a cuarto año del nivel secundario, el Diseño Curricular establece que: se debe trabajar sobre una característica fundamental, que es la completitud del campo numérico de los irracionales; se debe enseñar que la raíz cuadrada de cualquier número natural que no sea cuadrado perfecto no es una operación a resolver. Es un número y es, además, la única forma de escribir ese número exactamente; la operatoria debe ser trabajada con cálculos sencillos, que los alumnos puedan razonar y reflexionar, evitando extensos cálculos mecanizados con escaso valor matemático; es conveniente trabajar con las definiciones y propiedades de las operaciones que con la memorización de procedimientos para extraer factores o racionalizar de forma mecánica; el empleo de calculadoras científicas para trabajar con números irracionales debe dar lugar a la discusión de procedimientos y resultados.

En relación con los dos años posteriores de la escolaridad secundaria, los números irracionales son aplicados en operaciones con intervalos en $\mathrm{R}$, logaritmos y en la ampliación del campo numérico a los números complejos.

El Diseño Curricular también indica que, a pesar de que la Matemática escolar difiere del trabajo científico, en el aula se pueden y deben vivenciar el estilo y las características de la tarea que realiza la comunidad matemática. De esta forma se invita a los estudiantes a percibir a la disciplina como un quehacer posible para todos. Agrega que por lo general la Matemática escolar se caracteriza por una profusión de definiciones abstractas, procedimientos mecánicos, desarrollos unívocos y acabados, y demostraciones formales junto con un uso apresurado de la simbología. Esto contribuye a la creencia de que las personas que no son capaces de asimilar los conocimientos que se vinculan a ella de modo sistemático, en el orden y la cantidad en la que se presentan, fracasan por falta de capacidad para la materia.

El Diseño Curricular propone un cambio respecto del posicionamiento del docente, considerando que debe abandonar el lugar central que históricamente ha tenido dentro del aula para ocupar otro espacio en la dinámica de la clase; espacio que permita a los jóvenes interactuar con sus pares y con la propuesta de trabajo. Sin embargo, el encuentro de los alumnos con las propuestas que se planifiquen no garantiza por sí mismo que ellos aprendan Matemática. La intervención del docente es fundamental para que el aprendizaje sea posible y debe responder a estrategias que trasciendan la exposición como única dinámica de clase.

\section{Metodología}

Esta investigación emplea un enfoque metodológico cualitativo para analizar las contribuciones que realiza en el aprendizaje de los números irracionales la puesta en escena de una propuesta didáctica con un enfoque histórico. El alcance del estudio es descriptivo. La intención es caracterizar la manera en que los alumnos desde la acción y desde su reflexión construyen su propio conocimiento, estudiando cómo influye en el aprendizaje el intercambio con sus pares y el aporte de un enfoque histórico en la construcción de la noción de número irracional. El tipo empleado se corresponde con un estudio de caso, eligiendo como tal a cuarto año de la Escuela Agraria de la ciudad de Pergamino, para conocer la incidencia de una propuesta didáctica sobre números irracionales con un enfoque histórico.

El año de la escolaridad secundaria del presente estudio (cuarto) fue seleccionado debido a que los alumnos han realizado, en los años anteriores, un recorrido exhaustivo de los conjuntos numéricos. Para que los estudiantes puedan comprender el significado de los números irracionales, es necesario que tengan en claro el concepto de número racional (trabajado en primer, segundo y tercer años) y la diferenciación de este con los números naturales y enteros. Además, la selección de este año de la escolaridad se debe a que los números irracionales constituyen uno de los contenidos curriculares previstos para su enseñanza en cuarto año de la educación secundaria obligatoria, de acuerdo a la selección de contenidos establecida en el Diseño Curricular.

Los participantes de la investigación son todos los alumnos de una división de cuarto año de la Escuela Agraria de Pergamino. Son 35: 19 varones y 16 mujeres, entre los cuales hay cuatro repitentes. Sus edades oscilan entre los 15 y los 16 años. En el marco de la investigación, se constituyeron 10 grupos, de los cuales trabajaron ocho. En su diagnóstico el profesor manifiesta que es un curso donde es muy difícil lograr el orden necesario para trabajar con tranquilidad. Suelen no cumplir con las tareas extraescolares y la mitad aproximadamente viene reprobando la materia. La inasistencia también es una cuestión importante, ya que por clase se ausentan en promedio cinco alumnos. En 
cuanto al nivel socioeconómico, se observa que es un grupo heterogéneo: un poco menos de la mitad proviene de clase media y el resto, baja; aunque no se observan casos de marginación.

Considerando la clasificación de Bravin y Pievi (2008), el diseño de la investigación es cuasi experimental, ya que se aplica una propuesta didáctica intencionalmente diseñada a un grupo de estudiantes ya conformado. Se realiza un estudio longitudinal, debido a que se analiza el fenómeno durante un período de tiempo (16 clases; desarrolladas en dos meses) en el que interesa el proceso que se da al implementar la propuesta didáctica sobre números irracionales con un enfoque histórico. La investigación adopta un carácter eminentemente empírico, donde los datos para la elaboración de los resultados provienen de la experiencia en el campo situado de indagación.

Para la recolección de la información, la técnica que se implementa es la observación de clases. Se toman notas de campo para facilitar un posterior estudio y reflexión sobre los acontecimientos sucedidos en el aula. También se emplean grabaciones en audio y video, para obtener un registro permanente que facilite una continua revisión de los hechos. Para el procesamiento de la información, se analizan las respuestas escritas de cada una de las actividades resueltas por los grupos de alumnos, mediante la técnica de análisis de contenido (Ander-Egg, 2003). También se examina, mediante esta técnica, el registro que el docente realiza sobre sus apreciaciones durante la implementación de la propuesta didáctica así como las notas de campo de la observación de clases efectuada.

El instrumento empleado es una propuesta didáctica que toma a la Historia de la Matemática como un recurso a tener en cuenta para el aprendizaje, en este caso, de los números irracionales (ver Anexo). La misma consta de 11 actividades distribuidas en seis etapas, de acuerdo a lo que se les solicita a los estudiantes. La intención es que se constituya en una alternativa de enseñanza orientada a generar en los alumnos un aprendizaje activo, participativo y reflexivo. Por ello se invita a los estudiantes a recorrer un camino similar a como lo hicieron los matemáticos de la época hasta obtener el descubrimiento de los números irracionales. Es imprescindible el acompañamiento y la motivación del docente durante el desarrollo de estas actividades, para que los alumnos puedan vivenciar la incertidumbre que les produjo a los matemáticos de la época el hallazgo de estos números.

Las categorías de análisis quedan delimitadas por las cuatro perspectivas (histórica, educativa, matemática y curricular) oportunamente presentadas.

En la Tabla 1 se detalla/n la/s actividad/es que cada etapa de la implementación de la propuesta didáctica comprendió así como la cantidad de clases destinada a cada etapa.

Tabla 1. Actividades y clases para cada etapa de la propuesta

\begin{tabular}{ccc}
\hline Etapa & Actividad/es & Cantidad de clases \\
\hline 1 & 1 & 3 \\
\hline 2 & 2 & 2 \\
\hline 3 & 3 & 2 \\
\hline 4 & 4 y 5 & 2 \\
\hline 5 & 6 a 10 & 6 \\
\hline 6 & 11 & 1 \\
\hline
\end{tabular}

\section{Resultados}

Se resumen aquí los hallazgos en función a los interrogantes de la investigación presentados en el primer apartado. Para responder a ¿Cómo construyen los alumnos el significado de número irracional cuando las clases se desarrollan siguiendo una propuesta didáctica con un enfoque histórico?, se analizan las distintas etapas de trabajo en la implementación de la propuesta didáctica.

Primera etapa: En la actividad 1 los alumnos trabajan en el origen prehistórico de los campos numéricos conocidos por ellos y los fundamentos de la creación de los mismos. La intención es que puedan aproximarse al valor que la Historia tiene en el surgimiento de los conocimientos matemáticos. La resolución de esta actividad les resulta dificultosa, debido a que desconocen esta metodología de trabajo. El hecho de relacionar la Historia con los contenidos matemáticos produce desorganización y desconcierto en algunos estudiantes. Se observa apuro en dar respuesta, sin considerar la necesidad de un tiempo de lectura acorde para su elaboración. Por momentos es posible apreciar en los alumnos la necesidad de que la tarea resulte más sencilla, asemejándose a la resolución de ejercicios en los que aplican reglas prácticas aprendidas.

Sequnda etapa: Luego, para guiar a los estudiantes hacia el nuevo campo numérico, se les presenta un trabajo matemático que desencadena una problemática, la misma que vivieron los matemáticos de esa época: el cálculo de la 
longitud de la diagonal del cuadrado unitario. Los grupos construyen el cuadrado y calculan la diagonal. Aquí surge la inquietud y el intercambio de opiniones debido al resultado obtenido: $\sqrt{2}$, un número desconocido hasta el momento. En un primer intento los alumnos buscan redondear el resultado obtenido de calcular la raíz cuadrada de 2, lo que provoca confusiones, ya que lo asocian a los racionales. Seguidamente los estudiantes descubren con una mínima intervención del docente que $\sqrt{2}$ no pertenece al campo de los números racionales, ya que no pueden expresarlo como fracción. Los alumnos se encuentran con el mismo problema que los pitagóricos: el hallazgo de un número que no pertenece a ningún campo numérico conocido. Manifiestan actitudes de desconcierto, mucho asombro y el deseo de conocer de qué campo numérico se trata.

Tercera etapa: Los estudiantes trabajan en la demostración que los pitagóricos hicieron de la irracionalidad de $\sqrt{2}$, lo que les permite afianzar la conclusión obtenida en la etapa anterior: "Si los números tienen infinitas cifras decimales no periódicas, no pueden ser expresados como fracción”. En un diálogo que los distintos grupos mantienen con el docente este les pregunta cómo se llama el campo numérico al que pertenece dicho número. Solo un grupo responde diciendo "los irracionales" y, ante el pedido de explicación del significado de esta palabra, el grupo dice que no tiene razón de ser, como si se tratase de algo disparatado. El docente guía a los alumnos mediante el intercambio de opiniones para que puedan emitir la definición de número irracional. Concluyen que son aquellos que no pueden ser expresados como fracción y que tienen infinitas cifras decimales no periódicas. Los estudiantes logran entender, interpretar y construir el significado de número irracional cuando vivencian el encuentro con $\sqrt{2}$, al medir la diagonal del cuadrado unitario. Llegado este momento, así como les sucedió a los pitagóricos, se produce en cada uno de los alumnos una ruptura que les permite ampliar sus conocimientos, descubriendo un nuevo campo numérico: el de los números irracionales. Se va renunciando a la necesidad de transformarlos en expresiones decimales (mediante la resolución de la operación) para reconocerlos como números en sí mismos.

Cuarta etapa: Averiguan acerca del significado de una tablilla de arcilla, de la cual interpretan el método empleado por algunos matemáticos para obtener un valor aproximado de $\sqrt{2}$. Luego realizan algunos cálculos, para consolidar procedimientos asociados al concepto y que, por lo tanto, lo nutren. Dichos cálculos son, a su vez, al estilo de la época.

Quinta etapa: Se da lugar a una actividad de ampliación, en la cual los alumnos exploran otros números irracionales. De esta manera extienden el abanico de conocimientos respecto de este campo numérico desde un plano matemático (no solo raíces de números primos, como hicieron hasta ahora), sino también desde un plano extra-matemático, para robustecer la significación de este conjunto numérico.

Sexta etapa: Realizan una metacognición de lo transitado, tanto a nivel conceptual (englobando la perspectiva "matemática") como a nivel metodológico (englobando la perspectiva "histórica").

En cuanto a ¿Cuáles son los aspectos favorables de la propuesta didáctica para que los alumnos construyan el significado de número irracional?, fue posible advertir varios.

Los alumnos logran interpretar el concepto de número irracional a partir de una ruptura con los racionales y de cierto desconcierto inicial. Se sorprenden acerca del método babilónico empleado para la aproximación de la raíz cuadrada de un número. No pueden entender cómo con tan pocos recursos antiguamente podían descubrir semejantes cosas. Para ellos esos números siempre estuvieron guardados en la calculadora, como si esta hubiese sido la creadora. También manifiestan asombro por las innumerables aplicaciones que tienen los números irracionales. Una de ellas responde a la armonía del cuerpo humano, les llama la atención saber que, desde la antigüedad, la belleza y armonía corporal responden al número de oro.

Por otro lado, al ser consultados, siete de los ocho grupos consideran que aprender Matemática con su Historia es muy significativo, ya que les permite conocer cómo fueron evolucionando los conocimientos y no como el producto final de "algo" a lo que no le encuentran sentido. En algunos casos agregan que por momentos se olvidaban de la tensión que les suele provocar estar en una clase tradicional de Matemática, dado que el clima en la resolución de las consignas era agradable y sin presiones. Manifiestan que trabajar de este modo les permite fortalecer las capacidades individuales mediante la interacción con sus compañeros de grupo. La reflexión, crítica e intercambio de opiniones los invita a razonar antes de dar una respuesta. En algunos casos consideran que la forma de trabajo empleada los ayudó a acercarse al docente para consultar sin prejuicios, situación que no les resulta habitual durante clases tradicionales de Matemática. De hecho reconocen a la estrategia didáctica empleada como productiva para aquellos alumnos a los que les cuesta Matemática, ya que se abandona la rigidez y se da lugar a la flexibilidad en el aprendizaje. En síntesis, expresan que mediante esta metodología se sienten partícipes en la construcción de aprendizajes porque permanentemente trabajan, seleccionan información, comparan y analizan.

Finalmente, otro aspecto a mencionar es la fortaleza de las actividades seleccionadas en la propuesta, que propendieron a fomentar la construcción del significado de número irracional mediante un enfoque histórico, así como el compromiso del docente en el abordaje de cada una de las actividades, ya que muchas veces resulta más fácil llenar el pizarrón de ejercicios que trabajar con esta metodología.

Acerca de ¿Cuáles son los aspectos desfavorables?, también advertimos algunos.

En ocasiones hubo dificultades entre los compañeros de grupo debido a la falta de compromiso en la búsqueda de material para resolver las actividades así como en el hallazgo correcto del material en Internet, ya que les aparece 
mucha información y les cuesta decidir cuál es pertinente.

También a veces se apreciaron actitudes de poca paciencia ante la posibilidad de emitir una respuesta. El impulso inicial de los estudiantes es resolver las actividades como un cálculo aritmético, pero este tipo de trabajo comprende un proceso que demanda tiempo y dedicación.

A nivel institucional, algunas clases son interrumpidas por otras tareas (feria de ciencias, limpieza del tanque de agua) que provocan una discontinuidad en el trabajo. Para retomarlo, se requiere una participación mayor del docente.

A nivel curricular, debido a los contenidos que le faltaban desarrollar al profesor para atender a lo prescripto, se decide dedicar menos tiempo de puesta en común en las clases a partir de la cuarta.

\section{Conclusiones}

A partir de la experiencia hemos evidenciado que los alumnos, en ciertas ocasiones, pueden "ponerse" en el lugar de los grandes matemáticos que trataron el tema, aunque es inevitable que en algunos casos les cueste encontrar ese lugar, ya que hay contenidos, palabras y actividades que no llegan a interpretar por su poca experiencia en el estudio de la Matemática. En algunos casos los alumnos que habitualmente no trabajan se han interesado en el tema y han realizado las actividades requeridas logrando mejorar su situación académica. La Matemática empleando un enfoque histórico constituye una buena herramienta a implementar en las clases de Matemática, posiblemente en algún contenido en especial durante el ciclo lectivo y no en todos (por los tiempos que requiere el currículum escolar). Trabajar contenidos acompañados de su desarrollo histórico colabora en los aprendizajes para que los estudiantes puedan encontrarle el sentido tantas veces reclamado.

Con respecto al supuesto de partida: "Aprender los números irracionales a través de un enfoque histórico contribuye a desarrollar en los estudiantes un espíritu crítico, que les permita valorar la importancia de este campo numérico y la significatividad de los mismos", se advierten indicios a favor del mismo. Los alumnos consideran, en su mayoría, que el aprendizaje de los números irracionales mediante dicho enfoque les permite conocer las raíces del surgimiento de este contenido, enriqueciéndolo y otorgándole la significatividad que el mismo merece. También ha sido posible observar involucramiento e inquietudes emergentes por parte de los estudiantes en las clases. El desarrollo de tal espíritu crítico en los estudiantes se condice con los principios de la EMC oportunamente presentados, al mismo tiempo que interpela la tradicional secuencia teoría-ejemplo-ejercicio sin mucho margen para reflexionar o cuestionar (Serna, Castañeda y Montiel, 2014) y repercute en la formación de valores (Gómez, Fernández y Kempf, 2006).

Se considera que la situación de sentimientos negativos planteada en la presentación del artículo pudo relativamente revertirse mediante la implementación de la propuesta didáctica. Los alumnos pudieron vivenciar la necesidad de la creación de este nuevo campo numérico, estudiar el proceso que se realizó para dar con este conocimiento así como la aplicación del mismo en innumerables situaciones relacionadas con la cotidianidad. Se encuentra así armonía con lo expresado por Wheeler (1980) y Lara y Sgreccia (2010), en cuanto a que enseñar Matemática con su Historia enriquece los procesos de enseñanza y aprendizaje, desarrollando nuevas habilidades en los alumnos, y mejorando su actitud hacia esta área del conocimiento.

Se refuerza de este modo lo señalado por de Guzmán (1992) y Jorge et al (2010) acerca de la importancia de la Historia de la Matemática en la configuración de los conocimientos del profesor y, más aún, cómo integrar la Historia de la Matemática en el conocimiento didáctico del profesor en Matemática (Mora, Guacaneme y Jiménez, 2016).

En esta propuesta también se involucró a las TIC como recurso para la búsqueda de material que permita dar con los conocimientos históricos necesarios para la resolución de las actividades, aspecto también de relevancia para la EMC. Al respecto cabe advertir que los estudiantes evidenciaron cierta debilidad en su aprovechamiento, pues se vieron abrumados debido a tanta información. Muchas veces los estudiantes hallaban contenidos desconocidos en sus búsquedas que igualmente ponían en sus respuestas sin poder entender a qué se estaban refiriendo realmente. Se considera oportuno para próximas implementaciones de la propuesta didáctica, sugerido tanto por los estudiantes como por el docente, una previa preparación del material histórico que los alumnos deben analizar para dar respuesta a las consignas de trabajo, mediante por ejemplo Webquest.

El trabajo realizado ha sido una experiencia enriquecedora que ha respondido a los interrogantes planteados otorgando a nuestra experiencia docente una nueva mirada con respecto a la enseñanza de la Matemática. Hemos podido evidenciar que contemplar la Historia de la Matemática en la enseñanza de esta disciplina es una muy buena alternativa a tener en cuenta. Tanto los alumnos como el propio docente les encuentran sentido a los contenidos matemáticos con los que interactúan, que en un principio pueden parecer tan alejados de la cotidianidad como los números irracionales. Así, alimentan su deseo de conocer, de desarrollar sus conocimientos matemáticos, su pensamiento crítico, su valoración hacia la ciencia y humanidad. Esta propuesta ha intentado sembrar una semilla hacia esa búsqueda.

\section{Referencias bibliográficas}

Anacona, M. (2003). LaHistoria de las Matemáticas enlaEducación Matemática. Ema, 8(1), pp. 30-46.

Ander-Egg, E. (2003). Métodos y Técnicas de Investigación Social IV. Técnicas para larecogida de datos e información. Buenos Aires: 
Lumen.

Babini, J. (1966). Arquímedes: El Método. Buenos Aires: EUDEBA.

Bell, E. (1985). Historia de las Matemáticas. México: Fondo de Cultura Económica.

Boyer, C. (1986). Historia de la Matemática. México: AlianzaUniversidad Textos.

Bravin, C. y Pievi, N. (2008). Documento metodológico orientador para lainvestigación educativa. Buenos Aires: ME.

Dirección General de Cultura y Educación de laProvincia de Buenos Aires. (2009). Diseño curricular para laEducación Secundaria.

Buenos Aires: ME.

Freire, P. (2000). LaEducación como práctica de lalibertad (10ª ed.). Madrid: S.XXI.

Gómez, B. (2011). Marco preliminar para contextualizar lainvestigaciónenhistoria y educación matemática. Épsilon, 28(1), pp. 9-22.

Gómez, M.; Fernández, D. y Kempf, I. (2006). ¿De qué forma puede ser usada laHistoria de la Matemática como herramientadidáctica? ActaLatinoamericana de Matemática Educativa, 19, pp. 246-252.

González, P. (2004). LaHistoria de las Matemáticas como recurso didáctico e instrumento para enriquecer culturalmente suenseñanza. Suma, (45), pp. 17-28.

Guzmán, M. de (1992). TendenciasInnovadorasenEducación Matemática. Buenos Aires: OMA.

Higginson, W. (1980). On the Foundations of Mathematics Education.For the Learning of Mathematics, 1(2), pp. 3-7.

Jorge, F., Paixão, F., Cabrita, I. (2010).Contributos para a integração da história da matemática na formação inicial de professores. EnM.M. Moreno, A. Estrada, J. Carrillo y T.A. Sierra (eds.). InvestigaciónenEducación Matemática XIV (pp.365-377). Lleida: SEIEM.

Lara, E. y Sgreccia, N. (2010). Nepohualtzitzin: un modelo matemático de cualidad. Revista Latinoamericana de Etnomatemática, $3(2)$, pp. 24-54.

Mora, D. (2005). Didáctica critica, educación critica de las matemáticas y etnomatemática. La Paz: Campo Iris.

Mora, L.; Guacaneme, E. y Jiménez, W. (2016). Unejemplo de integración de laHistoria de las Matemáticas en el conocimientodidáctico de profesores de Matemáticas. Unión, (47), pp. 192-206.

Sánchez, J.C. y Valdivé, C. (2014). Estudiodel número irracional enloslibros de texto escolares: una visión desde el PMA. Premisa, 16(62), pp. 36-48.

Serna, L.; Castañeda, A. y Montiel, G. (2014). Aportes de lahistoria para el desarrollo de una situacióndidáctica para la tangente variacional. Premisa, 16(61), pp. 3-15.

Skovsmose, O. (1999). Hacia una filosofía de laEducación Matemática Crítica. (2a ed.). Bogotá: Una Empresa Docente.

Sorando, J.M. (2005). Matemáticas e Historia. Suma, (49), pp. 125-137.

Steiner, H. (1990). Needed Cooperation between Science Education and Mathematics Education. ZentralblattfurDidaktik Der Mathematik, 90(6), pp. 194-197.

Valero, P.; Andrade-Molina, M. y Montecino, A. (2015). Lo político enlaEducación Matemática: de laEducación Matemática Crítica a la Política Cultural de laEducación Matemática. Revista Latinoamericana de Matemática Educativa, 18(3), pp. 287-300.

Wheeler, D. (1980). Humanización de laEducación Matemática.Conceptos de Matemática, 55(1), pp. 7-14.

\section{ANEXO}

(1) "En la mente y en la acción del hombre prehistórico no están ausentes los números más simples, las formas más elementales y la ordenación más visibles de las cosas. En el hombre que da nombre a las cosas y a los actos; que conserva el fuego e imagina trampas para cazar animales; que construye viviendas y tumbas; que observa el movimiento de los astros y destaca direcciones espaciales; que computa distancias con su cuerpo y sus pasos; que graba escenas de un impresionante realismo; en ese hombre y en esas actividades están prefigurados los conceptos básicos de la Matemática: número, orden y medida".

Investiguen sobre el origen prehistórico de los campos numéricos.

¿Para qué se creó cada conjunto numérico?

¿Los conjuntos numéricos surgieron en el orden que hoy los estudiamos? ¿Por qué?

(2) En el S.VlaC comienza el período de esplendor de la civilización griega. En ese lugar y en ese momento nace la ciencia como hoy la entendemos, aparecen la abstracción y la generalización, ya que los pueblos más antiguos solo resolvían problemas referidos a casos particulares y concretos. Allí aparece la figura de Pitágoras. ¿Qué es lo primero que se nos ocurre hablar de él? Pitágoras, discípulo de Thales, es una figura legendaria; nació en Samos, isla griega en el mar Egeo, presuntamente en 585aC Viajó por Egipto y Babilonia donde accedió a los conocimientos matemáticos de esos pueblos. Luego se instaló en Crotona, antigua colonia griega que hoy es el sur de Italia, donde fundó una escuela en la que no se estudiaba solamente Matemática. Los pitagóricos eran místicos y funcionaban como una secta, a la que los que ingresaban entregaban todas sus pertenencias. Eran vegetarianos y tenían una característica revolucionaria para la época: permitían el acceso de mujeres. Sin embargo aquel que hiciera saber en el mundo exterior lo que pensaban y elaboraban recibía un castigo. Para los pitagóricos la belleza de la Matemática radicaba en la creencia de que los números (naturales) podían explicar todos los fenómenos de la naturaleza, y consideraban las fracciones como derivados de ellos. El número era el principio de todas las cosas; el lema de la escuela era: "Todo es número".

Profundicen sobre la vida y obra de Pitágoras.

Indaguen sobre otros matemáticos griegos contemporáneos de Pitágoras y sus aportes a esta ciencia. 
¿Los pitagóricos realizaron contribuciones a otras áreas?

¿Qué significado le otorgaban los pitagóricos a los números?

Imaginen que son griegos de hace 25 siglos. ¿Le darian un significado especial a algún número? ¿A cuál y por qué?

Dibujen un cuadrado y una de sus diagonales. Considerando que el lado del cuadrado mide una unidad y, aplicando el Teorema de Pitágoras, calculen el valor de la diagonal. ¿Pueden calcularlo con exactitud o no? ¿Por qué?

El número obtenido, ¿pertenece a algún campo numérico conocido? ¿Por qué?

(3) Los pitagóricos habían supuesto la existencia uno-uno entre las longitudes de los segmentos de líneas rectas y los números racionales.

Según el Teorema de Pitágoras, "en todo triángulo rectángulo el cuadrado de la hipotenusa es igual a la suma de los cuadrados de los catetos". De aquí se desprende que, si el triángulo es isósceles, la hipotenusa es inconmensurable, como obtuvieron en la actividad anterior. ¿Por qué se lo llama segmento inconmensurable? ¿Pueden expresar al número obtenido como un número racional?

Analicen e interpreten la siguiente demostración.

Se atribuye a los pitagóricos el descubrimiento de los números irracionales, hecho que les resultó perturbador y mantuvieron en secreto. Aristóteles menciona la demostración hecha por ellos, de que $\sqrt{2}$ es un número irracional, o sea, que no puede ser expresado como una razón entre dos enteros (se omite la demostración propiamente dicha por cuestiones de espacio en el artículo).

¿Es posible obtener un número irracional del cociente entre dos números enteros?

¿Cuántas cifras decimales no periódicas tiene un número irracional?

Escriban una definición de número irracional.

¿Cuál fue el origen histórico del término irracional?

Demuestren que $\sqrt{7}$ es un número irracional.

(4) ¿Qué es la tablilla Yale 7289 y qué información pueden encontrar en ella?

¿Por qué civilización fue desarrollada esta tablilla?

Ubiquen geográficamente la residencia de esta civilización, describan sus costumbres e indiquen los pueblos que la comprendían.

(5) Calculen $\sqrt{5}$ con el método babilónico. Utilicen todas las cifras decimales que aparezcan.

Comparen el resultado obtenido con el de la calculadora y observen cuántas cifras decimales obtuvieron.

Calculen, nuevamente con el método babilónico, $\sqrt{27}$. Realicen tres pasos, utilicen todas las cifras decimales que obtengan.

Calculen $\sqrt{27}$ en la calculadora y comparen resultados.

(6) Averigüen cómo se llama el número que obtuvimos al trabajar con la estrella de cinco puntas. ¿Qué características tiene? ¿Cuándo se lo descubrió?

Construyan un rectángulo áureo. Hallen la relación entre la base y la altura.

Investiguen sobre las proporciones en el cuerpo humano. Comprueben algunas con los integrantes del grupo.

Describan brevemente tres fenómenos cotidianos o naturales donde intervenga el número de oro.

(7) Redacten en forma procedimental el descubrimiento del número $\pi$ a partir de la actividad realizada en clase.

¿Qué número piensan que es? Ubíquenlo en algún campo numérico.

¿Quién lo descubrió y en qué época?

Analicen la poesía:

"Soy y seré a todos definible

mi nombre tengo que daros

cociente diametral siempre inmedible

soy de los redondos aros".

Cuenten las letras de cada una de las palabras de este poema y constaten con las cifras de qué famoso número coinciden.

¿Por qué se define como "cociente diametral siempre inmedible"?

¿A qué número alude la expresión: "Un famoso número que atrapó el interés de tantos matemáticos"? ¿Creen que fue el único que despertó tal interés, pueden citar otros?

Busquen información sobre Arquímedes y sus aportes a la Matemática.

¿Qué fracción utiliza Arquímedes para aproximar $\pi$ ? Encuentren los primeros siete decimales de su aproximación.

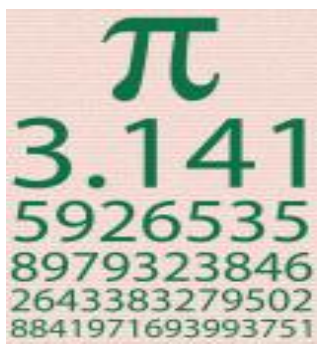

(8) Desde la invención de la rueda, en el período Neolítico, $\pi$ ha ocupado un lugar en la Historia. Veamos cómo se fue logrando una mejor aproximación.

\begin{tabular}{|l|l|l|}
\hline \multicolumn{1}{|c|}{ Quién lo obtuvo } & Año & \multicolumn{1}{c|}{ Valor } \\
\hline Egipto & $2000 \mathrm{aC}$ & 3,1605 \\
\hline China & $1200 \mathrm{aC}$ & 3 \\
\hline La Biblia & $550 \mathrm{aC}$ & 3 \\
\hline
\end{tabular}




\begin{tabular}{|l|l|l|}
\hline TsuChun-Chi & $500 \mathrm{aC}$ & $3,1415926<\pi<3,1415929$ \\
\hline Arquímedes & $300 \mathrm{aC}$ & $3,14084<\pi<3,142857$ \\
\hline Ptolomeo & $200 \mathrm{aC}$ & $377 / 120$ \\
\hline Aryabhata & $500 \mathrm{aC}$ & 3,1416 \\
\hline Bramaghupta & $600 \mathrm{aC}$ & $\sqrt{10}$ \\
\hline Fibonacci & 1220 & 3,141818 \\
\hline Lambert & 1766 & Nombra a $\pi$ irracional \\
\hline
\end{tabular}

Investiguen sobre Ptolomeo, Brahmagupta, Fibonacci y su relación con la Matemática.

En una línea de tiempo ubiquen estos distintos momentos en la Historia de $\pi$, utilizando una escala conveniente.

(9) Entre una de las referencias documentales más antiguas de $\pi$ se puede leer en la Biblia: "Hizo una fuente de metal fundido que media 10 codos de diámetro: era completamente redonda, y su altura era de 5 codos y una línea de 30 codos lo rodeaba" (I. Reyes 7,23). El interés aquí radica en que se utiliza: $\pi=3$.

Averigüen a cuántos centímetros equivale un codo.

El siguiente verso de Aryabhata, matemático hindú del S.IV, nos da la más antigua formulación sobre el valor aproximado de la relación que, más adelante, se llamará Pi.

"Sumar cuatro a cien, multiplicar por ocho, sumar todavía setenta y dos mil. Se obtiene así un valor aproximado de la circunferencia cuyo diámetro es de dos miríadas".

El texto indica que la longitud de esa circunferencia es $62832=\pi .20000$ ( $L=\pi . d$ y una miríada equivale a 10000 unidades) con lo que se deduce que el valor de $\pi$ utilizado es:

$\pi=\frac{\text { long.circ. }}{\text { diámetro }}=\frac{62832}{20000}=3,1416$ que es una muy buena aproximación, para el S.VI.

Hallen información sobre Aryabhata y sus aportes a la Matemática.

(10) Hay un número irracional muy importante en Matemática, que se lo usa en Economía, Química, Estadística y cálculo de probabilidades, como también en los pronósticos de crecimiento de población (humana, animal o vegetal).

Investiguen cuál es ese famoso número.

Indiquen el origen de su descubrimiento y los matemáticos implicados en el mismo.

Elijan una de las aplicaciones de este número y expliquen su utilidad.

(11) Definan con sus palabras qué es un número irracional. ¿En qué se diferencia de uno racional?

¿Por qué fue importante la creación de este campo numérico?

¿Creen que es importante aprender Matemática con su historia? ¿Por qué?

¿Cuáles son los aspectos positivos y negativos que han ido observando durante el desarrollo de cada una de las actividades?

De las actividades resueltas, ¿cuál fue la que más les atrajo? ¿Por qué?

¿Se sintieron partícipes de este nuevo aprendizaje? 